\title{
reciamuc
}

Revista cientifica de investigación actualización del mundo de las ciencias

Eduardo Patricio Padilla Manzano ${ }^{\text {a; }}$ Carolina Estefania Lema Tixi ${ }^{\text {b }}$

Diagnóstico y tratamiento de la cistitis aguda

Diagnosis and treatment of acute cystitis

Revista Científica de Investigación actualización del mundo de las Ciencias. Vol. 3

núm., 1, enero, ISSN: 2588-0748, 2018, pp. 272-285

DOI: 10.26820/reciamuc/3.(1).enero.2019.272-285

URL: $\underline{\text { http://reciamuc.com/index.php/RECIAMUC/article/view/235 }}$

Código UNESCO: 3205 Medicina Interna

Tipo de Investigación: Artículo de Revisión

Editorial Saberes del Conocimiento

Recibido: 10/12/2018

Aceptado: 20/01/2019

Publicado: 30/01/2019

Correspondencia: md.carolinalema@hotmail.com

a. Médico; Guayaquil, Ecuador; md.carolinalema@ hotmail.com

b. Médico; Guayaquil, Ecuador; eduardoppm17@hotmail.com 


\section{Diagnóstico y tratamiento de la cistitis aguda}

Vol. 3, núm. 1., (2019)

Eduardo Patricio Padilla Manzano; Carolina Estefania Lema Tixi

\section{RESUMEN}

Las infecciones del tracto urinario son las infecciones bacterianas más comunes en la mujer. La mayoría de las infecciones del tracto urinario son cistitis agudas no complicadas. Los identificadores de cistitis aguda no complicada son la frecuencia y la disuria en una mujer inmunocompetente en edad fértil que no tiene comorbilidades ni alteraciones urológicas. El examen físico es típicamente normal o positivo para la sensibilidad suprapúbica. Se recomienda un análisis de orina, pero no un cultivo de orina, para hacer el diagnóstico. Las pautas recomiendan tres opciones para el tratamiento de primera línea de la cistitis aguda no complicada: fosfomicina, nitrofurantoína y trimetoprima / sulfametoxazol (en regiones donde la prevalencia de la resistencia a la Escherichia coli no excede el 20 por ciento). Los antibióticos betalactamasa, amoxicilina / clavulanato, cefaclor, cefdinir y cefpodoxima no se recomiendan para el tratamiento inicial debido a las preocupaciones sobre la resistencia. Los cultivos de orina son recomendados en mujeres con sospecha de pielonefritis, mujeres con síntomas que no lo hacen resolver o que se repitan dentro de dos a cuatro semanas después de completar el tratamiento, y las mujeres que presentan síntomas atípicos.

Palabras Claves: Cistitis Aguda; Tratamiento; Infección de Tracto Urinario; Diagnostico. 


\title{
Diagnóstico y tratamiento de la cistitis aguda
}

Vol. 3, núm. 1., (2019)

Eduardo Patricio Padilla Manzano; Carolina Estefania Lema Tixi

\begin{abstract}
Urinary tract infections are the most common bacterial infections in women. Most urinary tract infections are uncomplicated acute cystitis. The identifiers of uncomplicated acute cystitis are frequency and dysuria in an immunocompetent woman of childbearing age who does not have comorbidities or urological changes. The physical examination is typically normal or positive for suprapubic sensitivity. A urinalysis, but not a urine culture, is recommended to make the diagnosis. The guidelines recommend three options for the first-line treatment of uncomplicated acute cystitis: fosfomycin, nitrofurantoin, and trimethoprim / sulfamethoxazole (in regions where the prevalence of Escherichia coli resistance does not exceed 20 percent). The antibiotics betalactamase, amoxicillin / clavulanate, cefaclor, cefdinir and cefpodoxime are not recommended for initial treatment due to concerns about resistance. Urine cultures are recommended in women with suspected pyelonephritis, women with symptoms that do not resolve or recur within two to four weeks after completing treatment, and women with atypical symptoms.
\end{abstract}

Key Words: Acute cystitis; Treatment; Urinary Tract Infection; Diagnosis. 


\section{Diagnóstico y tratamiento de la cistitis aguda}

Vol. 3, núm. 1., (2019)

Eduardo Patricio Padilla Manzano; Carolina Estefania Lema Tixi

\section{Introducción.}

Las infecciones del tracto urinario (ITU) son las bacterias más comunes en cuanto a infecciones en mujeres, con la mitad de todas las mujeres que experimentan al menos una ITU en su vida. La mayoría de las infecciones urinarias en las mujeres son la cistitis aguda no complicada causada por escherichiacoli (86\%), staphylococcussaprophyticus (4\%), especie Klebsiella (3\%), especie Proteus (3\%), especies de Enterobacter (1.4\%), especies de Citrobacter (0.8\%), o especies de enterococos (0.5\%) (Gupta, Scholes, \& Stamm, 1999).

A pesar de que la cistitis aguda no complicada puede no ser considerada como una condición grave, la calidad de vida de los pacientes a menudo se ve afectada significativamente. La cistitis aguda no complicada da lugar a una estimada de seis días de malestar principal a aproximadamente 7 millones de visitas clínicas por año con costos asociados de \$1.6 mil millones(Colgan, Keating, \& Dougouih, 2004 (24))

En un estudio de mujeres con cistitis aguda no complicada, casi la mitad de los participantes informaron que sus síntomas los causaron perder el trabajo o la escuela. Además, hasta la mitad de las personas con cistitis aguda no complicada también informó haber evitado la actividad sexual por un promedio de una semana.

Las infecciones del tracto urinario (ITU) se dividen en infecciones del tracto inferior y superior. Las infecciones del tracto inferior incluyen la vejiga y la uretra, mientras que las ITU superiores involucran los riñones. Muchas veces, la infección migra de una parte del tracto a otro. La mayoría de las infecciones urinarias son causadas por bacterias, aunque patógenos transmitidos sexualmente, micobacterias, los hongos y parásitos también dan lugar a tales 


\section{Diagnóstico y tratamiento de la cistitis aguda}

Vol. 3, núm. 1., (2019)

Eduardo Patricio Padilla Manzano; Carolina Estefania Lema Tixi

infecciones. Las infecciones urinarias bacterianas implican cualquier parte del tracto urinario y puede ser asintomático o se caracteriza por los síntomas que se presentan normalmente asociado con estas infecciones.

El diagnóstico se centra en el médico que toma una historia detallada de las quejas presentadas por el paciente, junto con un análisis de orina y cultivo de orina. El tratamiento usualmente involucra el uso de antibióticos. Cuando se considera adultos hasta 50 años, ITU bacterianas son mucho más comunes en las hembras, las incidencias de estas infecciones aumentan en pacientes masculinos y femeninos mayores de 50 años de edad. El tracto urinario es normalmente un medio ambiente estéril y esto es muy a menudo mantenido debido a varias razones que pueden incluir la acidez de la orina, el vaciado de la vejiga en micción y varias barreras inmunológicas y mucosas. Las infecciones urinarias ocurren cuando las bacterias patógenas ascienden por la uretra a la vejiga.

\section{Metodología.}

Para el desarrollo de este proceso investigativo, se plantea como metodología la encaminada hacia una orientación científica particular que se encuentra determinada por la necesidad de indagar en forma precisa y coherente una situación, en tal sentido (Davila, 2015) define la metodología "como aquellos pasos previos que son seleccionados por el investigador para lograr resultados favorables que le ayuden a plantear nuevas ideas". (p.66) 


\section{Diagnóstico y tratamiento de la cistitis aguda}

Vol. 3, núm. 1., (2019)

Eduardo Patricio Padilla Manzano; Carolina Estefania Lema Tixi

Lo citado por el autor, lleva a entender que el desarrollo de la acción investigativa busca simplemente coordinar acciones enmarcadas en una revisión bibliográfica con el fin de complementar ideas previas relacionadas al diagnóstico y tratamiento de la cistitis aguda a través de una revisión de literatura, para así finalmente elaborar un cuerpo de consideraciones generales que ayuden a ampliar el interés propuesto.

\section{Tipo de Investigación}

Dentro de toda práctica investigativa, se precisan acciones de carácter metodológico mediante las cuales, se logra conocer y proyectar los eventos posibles que la determinan, así como las características que hacen del acto científico un proceso interactivo ajustado a una realidad posible de ser interpretada. En este sentido, se puede decir, que la presente investigación corresponde al tipo documental, definido por Castro (2016), "se ocupa del estudio de problemas planteados a nivel teórico, la información requerida para abordarlos se encuentra básicamente en materiales impresos, audiovisuales y /o electrónicos”. (p.41).

En consideración a esta definición, la orientación metodológica permitió la oportunidad de cumplir con una serie de actividades inherentes a la revisión y lectura de diversos documentos donde se encontraron ideas explicitas relacionadas con los tópicos encargados de identificar a cada característica insertada en el estudio. Por lo tanto, se realizaron continuas interpretaciones con el claro propósito de revisar aquellas apreciaciones o investigaciones propuestas por diferentes investigadores relacionadas con el tema de interés, para luego dar la respectiva argumentación a los planteamientos, en función a las necesidades encontradas en la indagación. 


\section{Diagnóstico y tratamiento de la cistitis aguda}

Vol. 3, núm. 1., (2019)

Eduardo Patricio Padilla Manzano; Carolina Estefania Lema Tixi

\section{Fuentes Documentales}

El análisis correspondiente a las características que predomina en el tema seleccionado, llevan a incluir diferentes fuentes documentales encargadas de darle el respectivo apoyo y en ese sentido cumplir con la valoración de los hechos a fin de generar nuevos criterios que sirven de referencia a otros procesos investigativos. Para (CASTRO, 2016) las fuentes documentales incorporadas en la investigación documental o bibliográfica, "representa la suma de materiales sistemáticos que son revisados en forma rigurosa y profunda para llegar a un análisis del fenómeno".(p.41). Por lo tanto, se procedió a cumplir con la realización de una lectura previa determinada para encontrar aquellos aspectos estrechamente vinculados con el tema, con el fin de explicar mediante un desarrollo las respectivas apreciaciones generales de importancia.

\section{Técnicas para la Recolección de la Información}

La conducción de la investigación para ser realizada en función a las particularidades que determinan a los estudios documentales, tiene como fin el desarrollo de un conjunto de acciones encargadas de llevar a la selección de técnicas estrechamente vinculadas con las características del estudio. En tal sentido, (Bolívar, 2015), refiere, que es "una técnica particular para aportar ayuda a los procedimientos de selección de las ideas primarias y secundarias”. (p. 71).

Por ello, se procedió a la utilización del subrayado, resúmenes, fichaje, como parte básica para la revisión y selección de los documentos que presentan el contenido teórico. Es decir, que mediante la aplicación de estas técnicas se pudo llegar a recoger informaciones en cuanto a la revisión bibliográfica de los diversos elementos encargados de orientar el proceso de investigación. Tal como lo expresa, (Bolivar, 2015) "las técnicas documentales proporcionan las 


\section{Diagnóstico y tratamiento de la cistitis aguda}

Vol. 3, núm. 1., (2019)

Eduardo Patricio Padilla Manzano; Carolina Estefania Lema Tixi

herramientas esenciales y determinantes para responder a los objetivos formulados y llegar a resultados efectivos" (p. 58). Es decir, para responder con eficiencia a las necesidades investigativas, se introdujeron como técnica de recolección el método inductivo, que hizo posible llevar a cabo una valoración de los hechos de forma particular para llegar a la explicación desde una visión general.

Asimismo, se emplearon las técnicas de análisis de información para la realización de la investigación que fue ejecutada bajo la dinámica de aplicar diversos elementos encargados de determinar el camino a recorrer por el estudio, según, (Bolívar, 2015)las técnicas de procesamiento de datos en los estudios documentales "son las encargadas de ofrecer al investigador la visión o pasos que debe cumplir durante su ejercicio, cada una de ellas debe estar en correspondencia con el nivel a emplear" (p. 123). Esto indica, que para llevar a cabo el procesamiento de los datos obtenidos una vez aplicado las técnicas seleccionadas, tales como: fichas de resumen, textual, registros descriptivos entre otros, los mismos se deben ajustar al nivel que ha sido seleccionado.

\section{Resultados.}

La historia es la herramienta más importante para diagnosticar la cistitis aguda no complicada, y debe ser apoyada por un examen físico y análisis de orina. También es importante descartar una complicación más seria de ITU. Por definición, el diagnóstico dela cistitis aguda no complicada implica una IU no complicada en una mujer premenopáusica, no embarazada, sin anomalías urológicas ni comorbilidades conocidas. Los síntomas clásicos del tracto urinario inferior incluyen disuria, micción frecuente de pequeños volúmenes, y urgencia urinaria. Algunas veces puede ocurrir hematuria; el malestar suprapúbico es menos frecuente. La 


\section{Diagnóstico y tratamiento de la cistitis aguda}

Vol. 3, núm. 1., (2019)

Eduardo Patricio Padilla Manzano; Carolina Estefania Lema Tixi

probabilidad previa de la prueba de ITU en las mujeres es del 5\%; Sin embargo, cuando una mujer se presenta con el inicio agudo incluso de uno de los síntomas clásicos de la cistitis aguda no complicada, la probabilidad dela infección aumenta de 10 veces a 50 por ciento(Bent, Nallamothu, Simel, Fihn, \& Saint, 2002)

Por lo tanto, la presentación con uno o más síntomas puede verse como una prueba de diagnóstico valioso en sí mismo. Además, la probabilidad de la cistitis aguda no complicada es menor si el paciente informa de secreción o irritación vaginal, que son más probables en las mujeres con vaginitis o cervicitis. El nuevo inicio de frecuencia y disuria, con la ausencia de flujo vaginal o irritación, tiene un valor predictivo positivo de 90 por ciento para UTI.(Bent, Nallamothu, Simel, Fihn, \& Saint, 2002).

Un estudio prospectivo de 796 mujeres jóvenes sexualmente activa identificó factores de riesgo para ayudar a diagnosticar la ITU, incluidas las relaciones sexuales recientes, el uso del diafragma con espermicida, y ITU recurrentes (Hooton, Scholes, \& Hughes, 1996 Vol 7)

Examen físico y pruebas de diagnostico

El examen físico de pacientes concistitis aguda no complicada suele ser normal, excepto en el 10 a 20 por ciento de las mujeres con sensibilidad suprapúbica (Stamm, 1999). Se debe sospechar pielonefritis aguda si el paciente está mal y parece incómodo, especialmente si tiene fiebre concomitante, taquicardia o sensibilidad en el ángulo costo vertebral. La conveniencia y rentabilidad dela prueba de la tira reactiva de orina hace que sea una herramienta común de diagnóstico, y es una alternativa apropiada al análisis de orina y microscopía de orinapara diagnosticar la cistitis aguda no complicada. 


\section{Diagnóstico y tratamiento de la cistitis aguda}

Vol. 3, núm. 1., (2019)

Eduardo Patricio Padilla Manzano; Carolina Estefania Lema Tixi

Los nitritos y la esterasa leucocitaria son los indicadoresmas precisos de la cistitis aguda no complicada en mujeres sintomáticas. Para evitar la contaminación, lo conveniente es utilizar una muestra de orina limpia a mitad de camino paradiagnosticar la ITU, sin embargo, al menos dos estudiosno han mostrado diferencias significativas en el número de resultados contaminados o no confiablesentre especímenes recolectados con y sin limpieza preparatoria.

Los cultivos de orinase recomiendan solo para pacientes con sospecha de pielonefritis aguda; pacientes con síntomas que no se resuelven o que se repitendentro de dos a cuatro semanas después de la finalización del tratamiento; y pacientes que se presentancon síntomas atípicos. Un recuento de colonias mayor o igual a 103unidades por ml de un uropatógeno son diagnósticos de la cistitis aguda no complicada(Stamm, 1999). Sin embargo, los estudios han demostrado que más de 102coloniasformando unidades por ml en mujeres con los síntomas de una ITU representan un cultivo positivo. Los análisis de orina de rutina después del tratamiento o los urocultivos en pacientes asintomáticosno son necesario.

Otros estudios más allá del análisis de orina y cultivos de orina rara vez son necesarios para diagnosticar la cistitis aguda no complicada. Pacientes que presentan síntomas atípicos de cistitis aguda no complicada y aquellos que no responden a la terapia antimicrobiana adecuada puede requerir estudios de imagen, como tomografía computarizada o ecografía, para descartar complicaciones y otros trastornos.

\section{Tratamiento}

Ningún agente único se considera mejor para el tratamiento de la cistitis aguda no complicada y la elección entre los agentes recomendados deben ser individualizados(Gupta, 


\section{Diagnóstico y tratamiento de la cistitis aguda}

Vol. 3, núm. 1., (2019)

Eduardo Patricio Padilla Manzano; Carolina Estefania Lema Tixi

Hooton, \& Naber, International clinical practice guidelines for the treatment of acute uncomplicated cystitis and pyelonephritis in woman, 2011). Elegir un antibiótico depende de la efectividad del agente, los riesgos de efectos adversos, tasas de resistencia y propensión a causar daño colateral (es decir, efectos adversos de la terapia con antibióticos que pueden permitir la proliferación de organismos resistentes a los medicamentos, y la colonización o infección conorganismos multirresistentes). Además, los médicos deben considerar el costo, la disponibilidad y los factores específicos del paciente, como historial de alergia. En promedio, los pacientes comienzan a notar alivio de síntomas dentro de las 36 horas del inicio del tratamiento(Gupta, Scholes, \& Stamm, Increasing prevalence of antimicrobial resistance among uropathogens causing acute uncomplicated cystitis in woman, 1999).

Hay varios agentes de primera línea recomendados por la sociedad americana para las enfermedades infecciosas para el tratamiento de la cistitis aguda no complicada. Nueva evidencia apoya el uso de nitrofurantoína (macrocristales) y fosfomicina (Monurol) como terapia de primera línea(Gupta, Hooton, \& Naber, International clinical practice guidelines for the treatment of acute uncomplicated cystitis and pyelonephritis in woman, 2011) Los siguientes antimicrobianos representan el primer nivel:

1. Nitrofurantoína en una dosis de 100 mg dos veces al día durante cinco días;

2. Trimetoprima /sulfametoxazol (Bactrim, Septra) en una dosis de una tableta de doble concentración (160/800mg) dos veces al día durante tres días en regiones donde la prevalencia de resistencia de los uropatógenos comunitarios no exceda de 20por ciento

3. Fosfomicina en una sola dosisde $3 \mathrm{~g}$. 


\section{Diagnóstico y tratamiento de la cistitis aguda}

Vol. 3, núm. 1., (2019)

Eduardo Patricio Padilla Manzano; Carolina Estefania Lema Tixi

Tenga en cuenta que la duración de la terapia para la nitrofurantoína se ha reducido a cinco días.

En comparación con las anteriores pautas de la sociedad americana para enfermedades infecciosas de siete días, basado en investigaciones que muestran efectividad con una duración más corta de la terapia, la fosfomicina puede ser menos efectiva y no está ampliamente disponible en los Estados Unidos. Fluoroquinolonas (es decir, ofloxacina, ciprofloxacina y levofloxacina Levaquin)se consideran antimicrobianos de segundo nivel, y son apropiados en algunas configuraciones, tales como en pacientes con alergia a los agentes recomendados.

Aunque las fluoroquinolonas son efectivas, tienen la propensión a daños, y debe ser considerado para los pacientes con infecciones más graves que la cistitis aguda. Ciertos antimicrobianos (es decir, antibióticos beta-lactámicos, amoxicilina /clavulanato(Augmentin), cefdinir(Omnicef),cefaclor y cefpodoxime) pueden ser alternativas apropiadas si los agentes recomendados no se puede usar debido a la resistencia conocida o intolerancia al paciente. A pesar del amplio uso de productos de arándano para el tratamiento de las infecciones urinarias, no hay evidencia para apoyar su uso en pacientes sintomáticos.

\section{Resistencia antimicrobiana}

Los antibióticos betalactámicos no se recomiendan como tratamiento de primera línea para la cistitis aguda no complicada debido a la presencia generalizada de E. coli en tasas de resistencia superiores al 20 por ciento. La resistencia a las fluoroquinolonas generalmente se encuentra por debajo de10 por ciento en América del Norte y Europa, pero con una tendencia hacia el aumento de la resistencia en los últimos años(Gupta, Hooton, \& Naber, International 


\section{Diagnóstico y tratamiento de la cistitis aguda}

Vol. 3, núm. 1., (2019)

Eduardo Patricio Padilla Manzano; Carolina Estefania Lema Tixi

clinical practice guidelines for the treatment of acute uncomplicated cystitis and pyelonephritis in woman, 2011). Para preservar la efectividad de las fluoroquinolonas, no se recomienda como una opción de primer nivel. Fosfomicina y nitrofurantoína han retenido altas tasas de actividad in vitro en la mayoría de las áreas.

\section{Conclusiones.}

La cistitis es una patología muy común en mujeres que cursa con infección en la vejiga, cuyos síntomas son polaquiuria, tenesmo, disuria y dolor suprapúbico. El principal agente etiológico es E. coli, y está asociada a varios factores de riesgo, algunos evitables. El diagnóstico se confirma con una evaluación clínica, pero si hay riesgo de complicación se lleva a cabo un urocultivo. El tratamiento inicial es empírico, con antimicrobianos, por lo que es importante conocer la etiología de la enfermedad para elegir el más adecuado. En cistitis no complicadas se prefieren tratamientos cortos, mientras que en complicadas deben alargarse varios días. Existen diferentes medidas para prevenir la aparición de recidivas, siendo la más efectiva hasta el momento el uso de dosis subterapéuticas de antimicrobianos. El farmacéutico debe informar al paciente sobre cómo tomar la medicación para una correcta adhesión al tratamiento, así como informarle de los factores de riesgo asociados a cistitis y de cómo evitar la aparición de recidivas.

\section{Bibliografía.}

Bent, S., Nallamothu, B., Simel, D., Fihn, S., \& Saint, S. (2002). Does this woman have an acute uncomplicated urinary tract infection? JAMA, 2701-2710.

Bolívar, J. (2015). Investigación Documental. México. Pax.

Castro, J. (2016). Técnicas Documentales. México. Limusa. 


\section{Diagnóstico y tratamiento de la cistitis aguda}

Vol. 3, núm. 1., (2019)

Eduardo Patricio Padilla Manzano; Carolina Estefania Lema Tixi

Colgan, R., Keating, K., \& Dougouih, M. (2004 (24)). Survey of symptom burden in women with uncomplicated urinary tract infections. Clin Drug Investig., 55-60.

Davila, A. (2015). Diccionario de terminos cientificos. Caracas: Oasis.

Gupta, K., Hooton, T., \& Naber, K. (2011). International clinical practice guidelines for the treatment of acute uncomplicated cystitis and pyelonephritis in woman. Infectious Diseases Society of America and the European Society for Microbiology and Infectious diseases, 103-120.

Gupta, K., Scholes, D., \& Stamm, W. (1999). Increasing prevalence of antimicrobial resistance among uropathogens causing acute uncomplicated cystitis in woman. JAMA, 736-738.

Hooton, T., Scholes, D., \& Hughes, J. (1996 Vol 7). A prospective study of risk factors for symptomatic urinary tract infection in young women. $N$ Engl J Med, 468-475.

Stamm, W. (1999). Clinical Infectious Diseases: A Practical Approach. New York, NY: Oxford University Press. 